\title{
Rapamycin induces p53-independent apoptosis through the mitochondrial pathway in non-small cell lung cancer cells
}

\author{
NAOMI MIYAKE, HIROKI CHIKUMI, MIYAKO TAKATA, \\ MASAKI NAKAMOTO, TADASHI IGISHI and EIJI SHIMIZU
}

\begin{abstract}
Division of Medical Oncology and Molecular Respirology, Department of Multidisciplinary Internal Medicine, Tottori University, 36-1 Nishi-cho, Yonago-shi, Tottori-ken 683-8504, Japan
\end{abstract}

Received March 16, 2012; Accepted May 2, 2012

DOI: $10.3892 /$ or.2012.1855

\begin{abstract}
The mammalian target of rapamycin (mTOR) is a key kinase acting downstream of growth factor receptor PI3K and AKT signaling, leading to processes resulting in increased cell size and proliferation through translation control. Rapamycin, a specific inhibitor of mTOR, results predominately in G1 cell cycle arrest through translation control and occasionally, cell type-dependent apoptosis by an unknown mechanism. In this study, we investigated the effect and mechanism of action of rapamycin on non-small cell lung cancer (NSCLC) cell lines with p53 mutations. Cell proliferation was evaluated by modified MTT assay. The apoptotic effect of rapamycin was measured by caspase- 3 activation and flow cytometric analysis of Annexin $\mathrm{V}$ binding. The expression of $\mathrm{Bcl}-2$ and the release of cytochrome $\mathrm{c}$ from mitochondria were evaluated by western blotting. We found that rapamycin induced apoptosis in NSCLC cell lines with p53 mutations. Western blot analysis demonstrated that rapamycin downregulates the expression levels of Bcl-2, which leads to increased cytochrome c release from mitochondria and subsequent activation of caspase cascades. These findings suggest that rapamycin induces p53-independent apoptosis through downregulation of Bcl-2 and the mitochondrial pathway in NSCLC cell lines as a novel antitumor mechanism.
\end{abstract}

\section{Introduction}

Lung cancer remains the leading cause of malignancy-related mortality worldwide, in both men and women, with over a million cases diagnosed annually; non-small cell lung cancer (NSCLC) accounts for $\sim 85 \%$ of all lung cancer cases (1). Few patients are diagnosed at an early stage of NSCLC when curative resection

Correspondence to: Dr Hiroki Chikumi, Division of Medical Oncology and Molecular Respirology, Department of Multidisciplinary Internal Medicine, Faculty of Medicine, Tottori University, 36-1 Nishimachi, Yonago-shi, Tottori-ken 683-8504, Japan

E-mail: chikumi@med.tottori-u.ac.jp

Key words: rapamycin, apoptosis, non-small cell lung cancer, Bcl-2, cytochrome c is possible, and the objective response rate of advanced disease to chemotherapy is very low (2). Therefore, more effective and less toxic therapeutic agents are being sought for the treatment of NSCLC. The current state of knowledge has revealed that cancer cells have aberrant signaling pathways in cell cycle control, proliferation, invasion, and angiogenesis (3). Treatments targeted against these abnormal processes in cancer cells have shown promise in the management of lung cancer.

The phosphatidylinositol 3 kinase (PI3K)/serine-threonine protein kinase AKT pathway is a prototypic survival pathway that is constitutively activated in many types of cancer. This pathway is an attractive therapeutic target in cancer because: i) it is constitutively activated in many types of cancer; ii) it serves as a convergence point for many growth stimuli; and iii) it controls, through its downstream substrates, cellular processes that contribute to the initiation and maintenance of cancer cell proliferation. Moreover, the activation of the PI3K/AKT pathway confers resistance to many types of cancer therapy and is a negative prognostic factor for different tumor types (4-6).

mTOR, the mammalian target of rapamycin, is a wellpreserved serine/threonine kinase (MW, $289 \mathrm{kDa}$ ), with $95 \%$ of its amino acid identity conserved from yeast to human and mouse. It acts as a key kinase downstream of PI3K/AKT activation $(7,8)$. mTOR activation regulates downstream processes that ultimately result in increased cell size and proliferation through translational regulation by the downstream effectors: 4E-binding protein 1 and phosphorylated S6 kinase (p-S6k) (4). Several lines of evidence have suggested a critical role for mTOR in the development of lung cancer. For example, positive staining for phosphorylated mTOR was observed in $74 \%$ of human NSCLC tissue (9). Immunohistochemical staining for $\mathrm{p}-\mathrm{S} 6 \mathrm{~K}$, a marker for mTOR activation, has revealed that malignant progression from the normal lung, atypical alveolar hyperplasia (AAH), bronchoalveolar carcinoma, to adenocarcinoma was accompanied by progressively increasing levels of p-S6K (10). Therefore, mTOR is a promising molecular target for lung cancer.

The mTOR inhibitor rapamycin is a natural macrolide antibiotic produced by Streptomyces hygroscopicus, which binds to FKBP-12 (FK506-binding protein) and the resultant complex inhibits the protein kinase activity of mTOR. Rapamycin was originally discovered as a potent antifungal agent, but rapamycin and its derivatives have been explicitly designed and utilized for 
their effectiveness as anticancer agents, stemming from their superior solubility and stability properties (CCI-779, RAD001, and AP23573). Rapamycin and its derivatives CCI-779, RAD001, and AP23573 have been tested both as single agents and in combination with EGFR inhibitors in phase I and II clinical trials against several types of cancer, including $\operatorname{NSCLC}(6,11,12)$. The safety and efficacy of rapamycin and its derivatives in these clinical trials are indicative of the promising antitumor activity of these agents over a broad range of dosage levels.

Rapamycin and its derivatives have antitumor activities functioning through several mechanisms. As mTOR regulates the translation of mRNAs that encode protein required for $\mathrm{G} 1$ cell cycle progression and $\mathrm{S}$-phase initiation, mTOR inhibition of mTOR by rapamycin fundamentally results in cell cycle arrest. Although the effect of rapamycin for tumor cells in the clinical trials was thereby expected to be cytostatic, tumor regression was observed, indicating that rapamycin may also induce apoptosis. To date, reports on the ability of rapamycin to actually induce apoptosis vary from one type of cancer to another, and the mechanism by which rapamycin induces apoptosis in cancer cells is poorly understood. Rapamycin has been shown to induce apoptosis in BKS-2 immature B cell lymphoma (13), JN-desmoplastic small round cell tumors-1 cells (14), hepatocellular carcinoma cells (15), anaplastic large cell lymphoma (16), IGROV1 ovarian carcinoma cells (17), and rhabdomyosarcoma cells $(18,19)$. Conversely, rapamycin does not induce apoptosis in SU-DHL-4 B lymphoma cells (20). Regarding lung cancer cell lines, limited data are available, and they are inconsistent. Apoptosis was not induced in KLN-205 and A549 NSCLC cell lines by rapamycin alone (21), whereas apoptosis was induced in Calu6 NSCLC cells when treated with the combination rapamycin plus erlotinib (22).

The ability to induce apoptosis is an important component of an antitumor profile when further development of rapamycin and its derivatives are considered as anticancer agents, and when combined with other cytotoxic anticancer agents. Therefore, we examined the apoptotic ability of mTOR inhibitor rapamycin and its mechanism of action in NSCLC cell lines.

\section{Materials and methods}

Cell lines and culture conditions. The NSCLC cell line Lu99 was provided by the Cell Resource Center for Biomedical Research (Institute of Development, Aging and Cancer, Tohoku University, Sendai, Japan). The 86-2 cell line was provided by Dr S. Kobayashi (Miyagi Prefectural Semine Hospital, Miyagi, Japan) through the Cell Resource Center for Biomedical Research. The RERF-LC-AI lung cancer cell line was obtained from RIKEN cell bank (Tsukuba, Japan). The Ma10 and Ma25 cell lines were provided by Dr T. Hirashima (Osaka Prefectural Habikino Hospital, Osaka, Japan). Cells were cultured in DMEM (Wako, Osaka, Japan) or RPMI 1640 medium (Wako) containing $10 \%(\mathrm{v} / \mathrm{v})$ fetal bovine serum, penicillin (100 IU/ml), and streptomycin $(100 \mu \mathrm{g} / \mathrm{ml})$. Cells were grown at $37^{\circ} \mathrm{C}$ in humidified atmosphere with $5 \% \mathrm{CO}_{2}$. Cells from exponentially growing cultures were used in all experiments.

Preparation of mitochondrial and cytosolic extracts. Cells were collected by centrifugation at $300 \mathrm{xg}$ at $4^{\circ} \mathrm{C}$ for $5 \mathrm{~min}$ and then washed with ice-cold PBS. The cell pellets were resuspended in
Table I. Histological properties and p53 mutation status of nonsmall cell lung cancer cell lines.

\begin{tabular}{lll}
\hline Cell line & Histological sub-type & p53 status \\
\hline Ma10 & Adenocarcinoma & Mutant \\
Lu99 & Giant cell carcinoma & Mutant \\
$86-2$ & Large cell carcinoma & Mutant \\
Ma25 & Large cell carcinoma & Mutant \\
RERF-LC-AI & Squamous cell carcinoma & Mutant \\
\hline
\end{tabular}

MT buffer (250 mM sucrose, $20 \mathrm{mM}$ HEPES, pH 7.5, $10 \mathrm{mM}$ $\mathrm{KCl}, 1.5 \mathrm{mM} \mathrm{MgCl}_{2}$, 1 mM EDTA, $1 \mathrm{mM}$ EGTA, $1 \mathrm{mM}$ DTT, and $0.1 \mathrm{mM}$ PMAF), and homogenized. The homogenates were centrifuged at $10,000 \mathrm{x} \mathrm{g}$ at $4^{\circ} \mathrm{C}$ for $30 \mathrm{~min}$. The supernatant was used as the cytosol fraction, and the pellet was resuspended in MT buffer containing $0.1 \%$ Triton X-100 and $0.1 \%$ SDS as the mitochondrial fraction. The protein concentration of each fraction was determined by Bio-Rad Protein Assay kit (Bio-Rad, Hercules, CA, USA).

Western blot analysis. Cells were treated with various concentrations of drugs. Cell lysates were denatured in sample buffer containing SDS, and equal amount of total protein were separated on $15 \%$ SDS-PAGE and transferred to Immobilon-P (Millipore, Bedford, MA, USA) membranes. After blocking, the membranes were incubated with the following primary antibodies: anti-Bcl-2 (Cell Signaling Technology, Beverly, MA, USA), anti-cytochrome c (BD Biosciences Pharmingen, San Diego, CA, USA), and anti- $\beta$-actin (Sigma-Aldrich, St. Louis, MO, USA). The membranes were incubated with appropriate horseradish peroxidase-conjugated secondary antibodies, and detection was performed using ECL reagent (Amersham Biosciences, Amersham, UK).

Cell proliferation assay. Cellular proliferation assay was performed by modified MTT assay. Briefly, $1 \times 10^{4}$ cells were treated with rapamycin in flat bottom 96-well plates at different concentrations as indicated for $12,24,48,60$, and $72 \mathrm{~h}$. WST-8 (2-(2-methoxy-4-nitrophenyl)-3-(4-nitrophenyl)-5-(2,4-disulfophenyl)-2H-tetrazolium, monosodium salt) solution (Dojindo Laboratories, Co., Kumamoto, Japan) was added to each well and incubated for $2 \mathrm{~h}$ at $37^{\circ} \mathrm{C}$. The absorbance was measured at $450 \mathrm{~nm}$ using a Model 680 microplate reader (Bio-Rad). Cell viability was calculated as the mean absorbance of the wells containing treated cells divided by the mean for the untreated control wells. All experiments were performed at least in triplicate and were repeated 3 times.

Caspase activity assay. The activity of caspase-3 was determined by a caspase colorimetric assay kit (Medical \& Biological Laboratories, Co., Nagoya, Japan), according to the manufacturer's protocol. Briefly, rapamycin-treated cells were washed with ice-cold PBS and lysed in a lysis buffer. The cell lysates were tested for caspase-3 activities by incubating with a caspase-specific peptide conjugated to the molecule $p$-nitroaniline. The chromophore $p$-nitroaniline cleaved by caspase- 3 was 

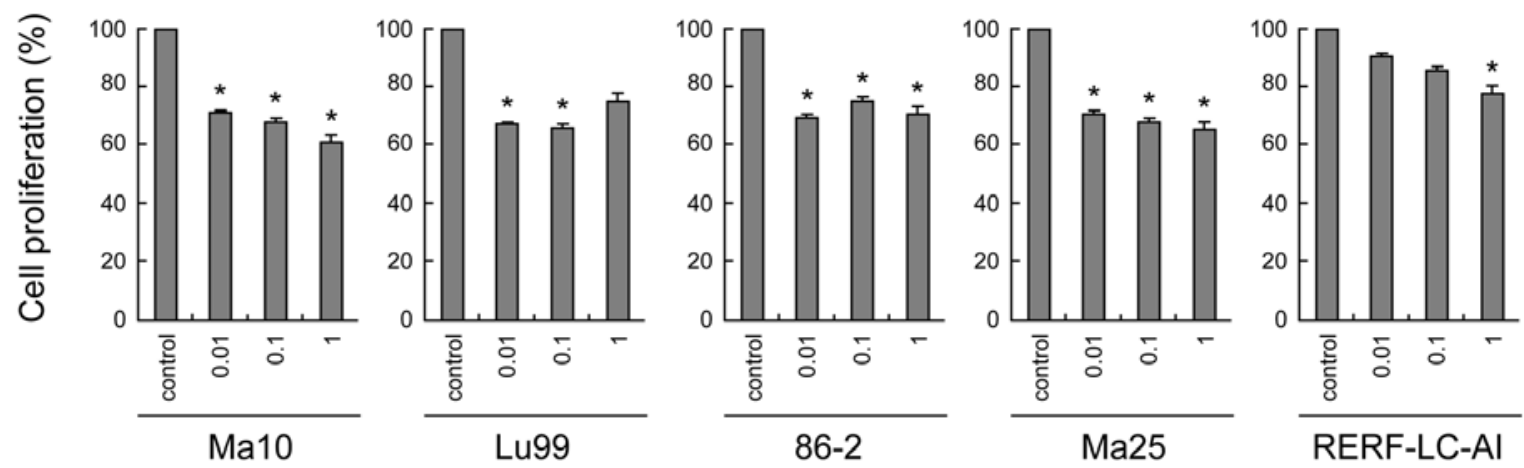

Figure 1. Continuous exposure of rapamycin suppresses the proliferation of p53 mutant NSCLC cell lines. Cells were continuously treated with the indicated concentration of rapamycin for $60 \mathrm{~h}$, and cell growth was determined by the WST- 8 assay. Error bars indicate SD of triplicate cultures. Data shown represent 3 independent experiments. ${ }^{*} \mathrm{P}<0.05$ versus placebo by Student's t-test.
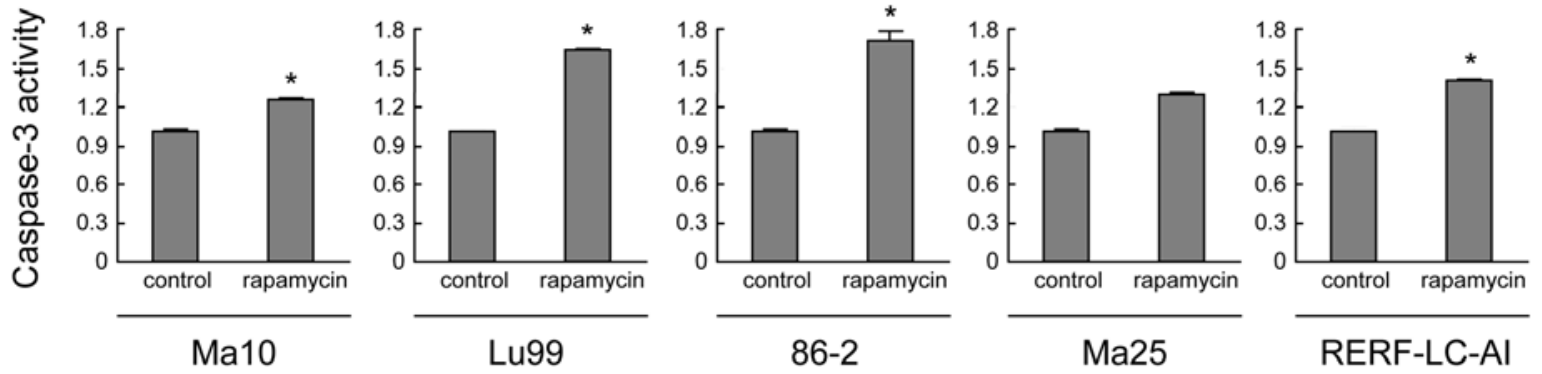

Figure 2. Effect of rapamycin on caspase-3 activity in NSCLC cell lines. Cells were treated with $1 \mu \mathrm{M}$ rapamycin for $48 \mathrm{~h}$, and caspase-3 activity was determined by a colorimetric method according to the manufacturer's instructions as described in Materials and methods. Bars are SD of triplicate cultures. Data shown represent 3 independent experiments with similar results. ${ }^{*} \mathrm{P}<0.05$ versus placebo by Student's t-test.

quantitated by measuring the absorbance at a wavelength of 405 nm with a microplate reader (Bio-Rad).

Apoptosis assay. Cells were harvested after incubation with or without $1 \mu \mathrm{M}$ rapamycin for up to 5 days and treated with $5 \mu \mathrm{l}$ of PE Annexin V solution and 7-amino-actinomycin D (7-AAD) (PE Annexin V Apoptosis Detection kit I, BD Biosciences Pharmingen). After washing, dual parameter flow cytometric analysis was performed to determine the percentage of apoptosis cells using a flow cytometer (FACSCalibur, BD Biosciences Pharmingen).

Statistical analysis. The statistical calculations were performed with SPSS 16.0 software (SPSS, Chicago, IL, USA). Student's t-test was used for a comparison between 2 groups, and $\mathrm{P}<0.05$ was considered statistically significant.

\section{Results}

Growth inhibitory effect of rapamycin on NSCLC cell lines with p53 mutation. To evaluate the ability of rapamycin to induce apoptosis without its confounding effects on cell cycle progression, we used 5 NSCLC cell lines with p53 mutation (Table I). The histological subtypes of lung cancer cell lines examined were adenocarcinoma (Ma10), giant cell carcinoma (Lu99), large cell carcinoma (86-2 and Ma25), and squamous cell carcinoma (RERF-LC-AI). Mutational status in p53 of these cell lines have been described (23-27). First, we examined the dose-dependent effect of rapamycin on the proliferation of these cell lines. As shown in Fig. 1, rapamycin generally suppressed the proliferation of all NSCLC cell lines, although the concentration required for significant suppression was different in each cell line. In most cell lines (Ma10, Lu99, 86-2, and Ma25 cells), very low concentrations $(0.01 \mu \mathrm{M})$ of rapamycin was sufficient for significant suppression of cell proliferation, whereas $1 \mu \mathrm{M}$ of rapamycin was required in RERF-LC-AI cells. These data suggest that rapamycin has a modest but obvious growth inhibitory effect on the growth of NSCLC cell lines that possess $p 53$ mutations.

Rapamycin induces apoptosis in NSCLC cell lines with p53 mutation. To evaluate the contribution of apoptosis to the growth-inhibitory effects of rapamycin, we then evaluated the apoptotic cell death induced by rapamycin. For this, we measured the activity of caspase-3, which is the most important effector caspase involved in rapamycin-induced apoptosis. As shown in Fig. 2, caspase-3 activities were significantly enhanced by rapamycin treatment of all NSCLC cell lines, except for Ma25 $(\mathrm{P}<0.05)$. These data suggest the potential ability of rapamycin to induce apoptosis in these lung cancer cell lines.

To further document the evidence of rapamycin-induced apoptosis, we then stained the cells with Annexin V-PE and the vital dye 7-AAD and analyzed the cells by flow cytometry after rapamycin treatment. Representative results are shown in Fig. 3A. In this analysis, cells that were primarily Annexin $\mathrm{V}$-negative and 7-ADD-negative were viable and not undergoing apoptosis (Fig. 3A, bottom left). Cells that stained positive for Annexin $\mathrm{V}$ and negative for 7-ADD were at an early stage of 


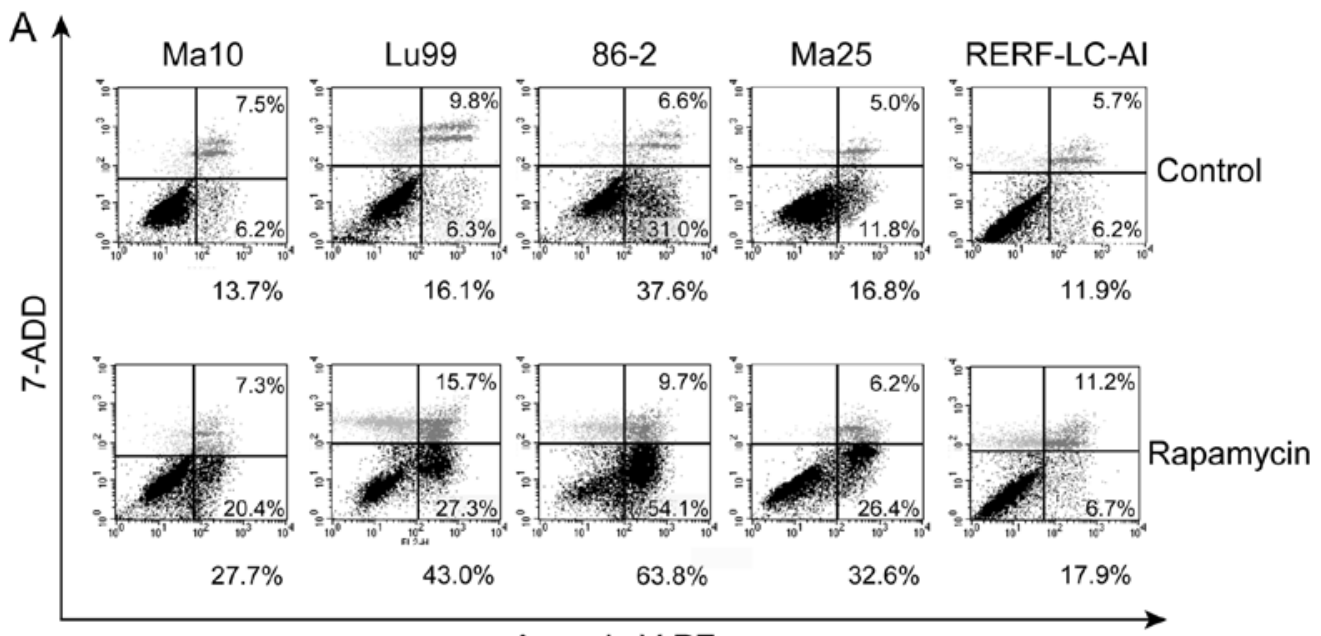

Annexin V-PE

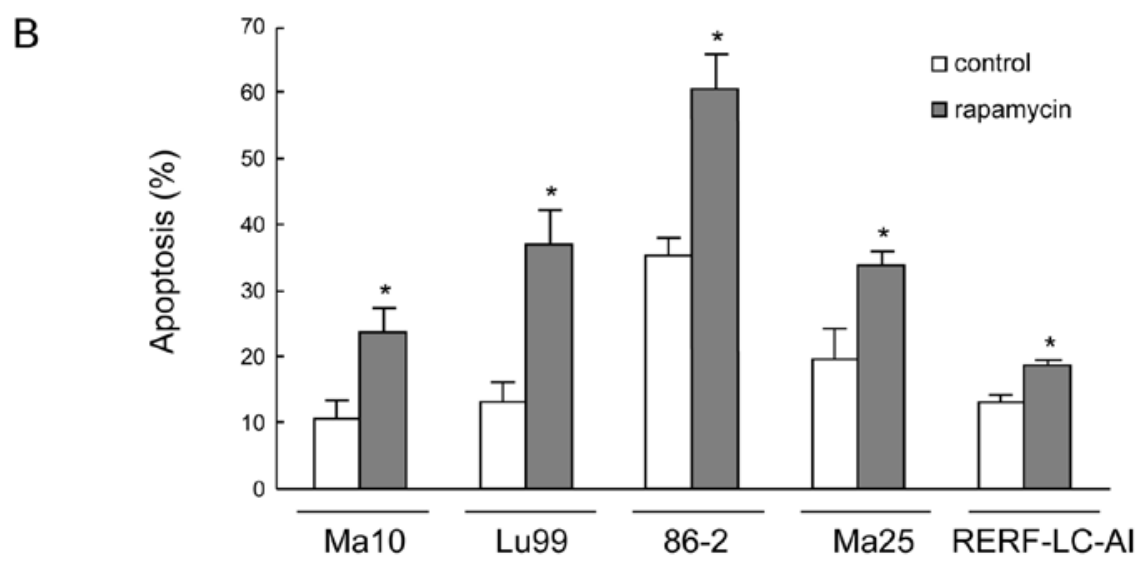

Figure 3. Rapamycin-induced apoptosis in NSCLC cell lines. Cells were treated with $1 \mu \mathrm{M}$ rapamycin for 5 days and harvested. Apoptosis was determined by flow cytometry as described in Materials and methods. (A) Representative data of dual staining for Annexin V-PE binding and 7-AAD uptake from 3 independent experiments. The percentage of Annexin V-positive and 7-ADD-negative cells that were in early-stage apoptosis (bottom right) and Annexin V-positive and 7-ADD-positive cells that were dead or in end-stage apoptosis (top right), are presented in each quadrant. The percentage of Annexin V-positive cells are presented at the bottom of each chart. (B) Summary of quantification of Annexin V-positive cells in a population of NSCLC cells treated with rapamycin. Data represent the summary of mean \pm SD from 3 independent experiments. " $\mathrm{P}<0.05$ versus placebo by Student's t-test.

apoptosis, in which the cell membrane integrity was present (Fig. 3A, bottom right). Cells that stained positive for both Annexin $\mathrm{V}$ and 7-ADD were either at the end stage of apoptosis, in the process of undergoing necrosis, or already dead (Fig. 3A, top right). For Ma10, Lu99, 86-2, Ma25, and RERF-LC-AI cells, early apoptotic cells in controls were 6.2, 6.3, 31, 11.8 and 6.2\%, respectively, and these frequencies represent an intrinsic apoptotic cell death property of each cell line. After 5 days of $1 \mu \mathrm{M}$ rapamycin treatment, for Ma10, Lu99, 86-2, and Ma25 cells, the frequency of apoptotic cells markedly increased to 20.4, 27.3, 54.1 and $26.4 \%$, respectively. In RERF-LC-AI cells, although the increase of early-stage apoptosis was slight $(\leq 6.7 \%)$, end-stage apoptosis and the percentage of dead cells (Annexin V-positive and 7-ADD-positive cells) were markedly increased from 5.7 to $11.2 \%$ by rapamycin treatment. Quantitative assessment of the percentage of Annexin V-positive cells from 3 independent experiments showed that apoptotic cell death was significantly increased by rapamycin treatment in all NSCLC cell lines (Fig. 3B).
Rapamycin suppresses Bcl-2 expression and promotes mitochondrial cytochrome c release. To explore the mechanism by which rapamycin induces apoptosis in $p 53$-mutated NSCLC cell lines, we examined the mitochondrial pathway of apoptosis. For this, we tested the expressions of 2 representative regulators of apoptosis in this pathway the, anti-apoptotic protein Bcl-2 and cytochrome $\mathrm{c}$ in the cytoplasm. As shown in Fig. 4A, the expression of Bcl-2 was markedly downregulated, and the expression of cytochrome $\mathrm{c}$ in cytoplasm was markedly upregulated by rapamycin treatment in a dose-dependent manner in all NSCLC cell lines. These data suggest that rapamycin induces apoptosis through $\mathrm{Bcl}-2$ and cytochrome $\mathrm{c}$.

Because $\mathrm{Bcl}-2$ acts to prevent the release of cytochrome $\mathrm{c}$ in mitochondria (28), we further examined the effect of rapamycin for these 2 proteins at the mitochondrial level using mitochondrial and cytosolic fractions. As shown in Fig. 4B, after treatment with $1 \mu \mathrm{M}$ rapamycin for $48 \mathrm{~h}, \mathrm{Bcl}-2$ levels in both mitochondrial and cytosolic fractions were decreased. Accordingly, the cytochrome c levels in the cytosol were significantly increased in all cell lines, while cytochrome $\mathrm{c}$ in mitochondrial fraction 

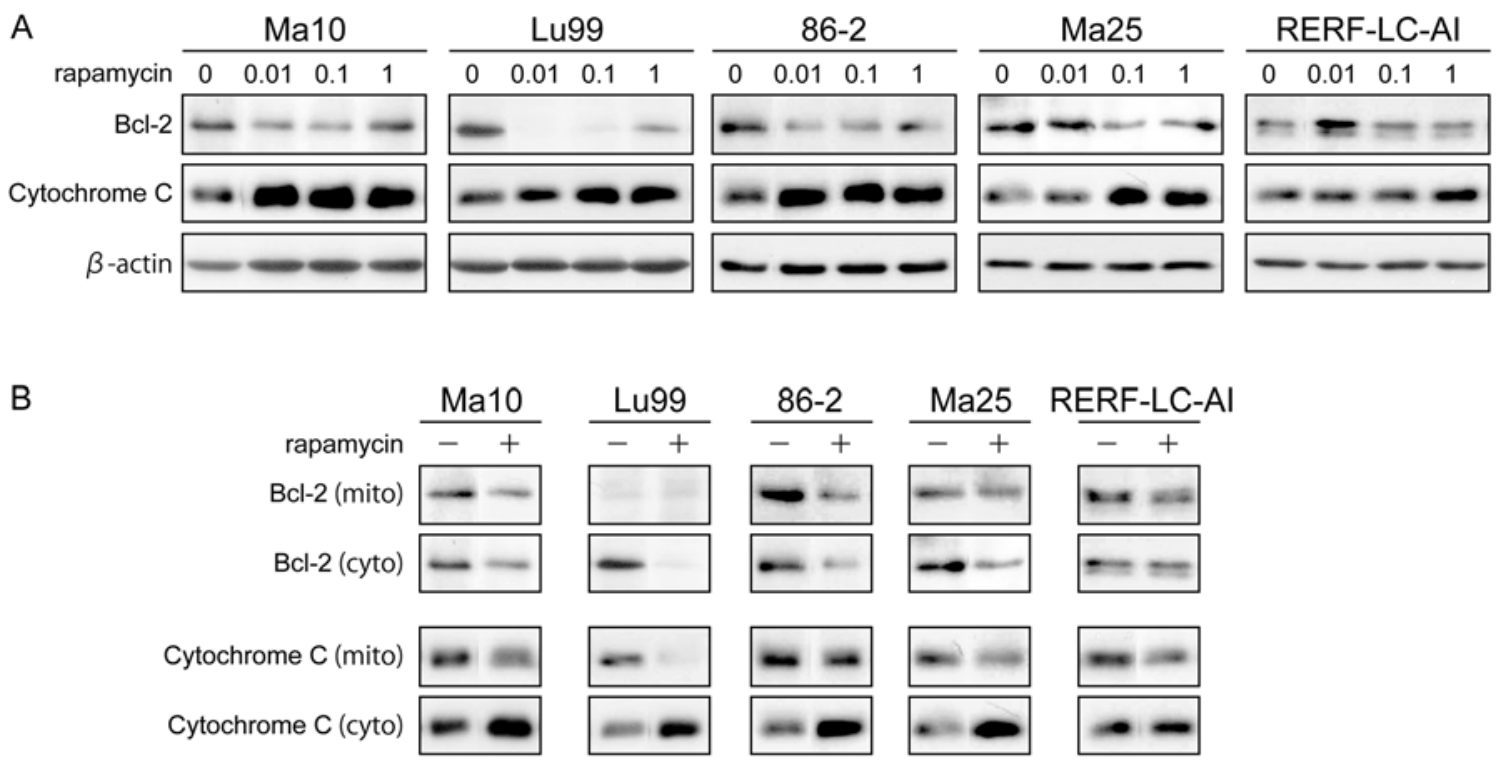

Figure 4. Western blot analysis of Bcl-2 and cytochrome c in rapamycin-treated NSCLC cell lines. (A) Cells were treated with increasing concentrations of rapamycin for $48 \mathrm{~h}$. Cytosolic fraction was prepared and analyzed by western blotting using specific antibodies targeting Bcl-2 and cytochrome c. An antibody for $\beta$-actin was used for measuring equal loading. (B) Cells were treated with $1 \mu \mathrm{M}$ rapamycin for $48 \mathrm{~h}$. After the preparation of mitochondrial and cytosolic fractions as described in Materials and methods, each fraction was analyzed by western blotting with indicated specific antibodies. Data represent 3 independent experiments with similar results.

decreased moderately to significantly. These data suggest that the mechanism that rapamycin utilizes to reduce $\mathrm{Bcl}-2$ expression levels in the cytoplasm, accompanied by decreased levels of $\mathrm{Bcl}-2$ in mitochondria, leads to the release of cytochrome $\mathrm{c}$ from mitochondria, and possible caspase activation.

\section{Discussion}

In the present study, we demonstrated the rapamycininduced apoptosis in NSCLC cell lines with p53 mutation. Mechanistically, we showed that rapamycin suppresses the expression level of Bcl-2, which leads to an increased release of cytochrome $\mathrm{c}$ from mitochondria and subsequent activation of caspase cascades. These findings suggest that the mTOR inhibitor possesses a novel mechanism for executing $p 53$-independent apoptosis in NSCLC cell lines.

Apoptosis induced by the inhibition of the PI3K/AKT pathway have been well documented, whereas the ability of mTOR inhibition to induce apoptosis and its potential mechanisms have not been consistently reported. AKT has been reported to phosphorylate and inhibit the function of the pro-apoptotic proteins Bad (29) and Bax (30), which subsequently execute antiapoptotic effects in cancer cells. Therefore, it is widely accepted that the inhibition of AKT induces apoptosis in various cancer cells. Conversely, regarding the inhibition of mTOR, the reported results are inconsistent across cell types, and the mechanisms in NSCLC cells remain unknown. For example, rapamycin has been reported to induce a cellular stress response characterized by rapid and sustained activation of the apoptosis signal-regulating kinase 1 (ASK1), leading to elevated phosphorylation of c-Jun and apoptosis in p53-mutated rhabdomyosarcoma cells (19). The relationship between cell cycle regulation and apoptosis has also been investigated in several studies. Aguirre et al reported that rapamycin blocks cell cycle progression in the G1/S phase by downregulating cyclin D1 and CDK4, followed by increased expression of caspase-3 and increased apoptotic cell death in a $p 53$ wild-type ovarian cancer cell line (17). In contrast, Huang et al reported continued G1 progression and S-phase entry resulting in a rapamycin-induced apoptosis in $p 53$-mutated rhabdomyosarcoma cell line (18). In the mitochondria, the key site of the apoptosis initiation, upregulated expression of the pro-apoptotic protein Bax, and downregulation of the antiapoptotic protein Bcl-XL by rapamycin have been described in small cell lung cancer (SCLC) cells (31), JN-DSRCT-1 cells (14), and HCC cells (15). Regarding Bcl-2, a well-recognized and important anti-apoptotic regulator of the mitochondrial pathway, downregulation by rapamycin, was described in SCLC (31), HCC (15), anaplastic large cell lymphoma (16), and rheumatoid synovial cells (32). Our data indicating that rapamycin downregulates Bcl-2 in NSCLC cell lines is consistent with the aforementioned studies in other types of cancer, with supporting evidence derived from the concomitant release of cytochrome $\mathrm{c}$ from mitochondria.

Our data showing that rapamycin can induce apoptosis in p53-mutated NSCLC cell lines is consistent with previous studies reporting that rapamycin induces apoptosis in p53-mutated cancer cells but not in $p 53$ wild-type cells (18). The only prior study testing the effect of rapamycin on NSCLC cells did not detect rapamycin-induced apoptosis in NSCLC cell lines with wild-type $p 53$ (21). One possible explanation for this discrepancy is that certain $p 53$ wild-type tumors harbor mutations that can suppress apoptosis downstream of $p 53$, and rapamycin cannot affect the anti-apoptotic function of these downstream effector molecules. Another possibility is that the anti-apoptotic property of cancer cells becomes dependent on proto-oncogene Bcl-2, but not on $p 53$, in $p 53$ mutated cancer cells. Therefore, the downregulation of Bcl-2 by rapamycin effectively induces apoptosis for these cell lines. In either instance, since the $p 53$ 
tumor suppressor gene is mutated in half of all cancer cells and is indirectly inactivated in many others, our finding that rapamycin induces apoptosis independently from $p 53$ is an important property, warranting further examination of its tumor specificity as an anticancer drug.

One limitation of this study, similar to prior studies that have reported on the downregulation of Bcl-2 by rapamycin $(15,16,31,32)$, is that the mechanism by which rapamycin downregulates $\mathrm{Bcl}-2$ has not been elucidated. Regarding the remaining Bcl-2 family member proteins, Tirado et al reported that the antiapoptotic protein Bcl-XL was downregulated by the inhibition of cap-dependent translation caused by the inhibitory action of rapamycin on mTOR (14). The pro-apoptotic protein Bax was upregulated by the action of rapamycin which prevents Bax degradation by mTOR-independent proteasome in tumor cells that have undetectable levels of Bcl-2 (14). Likewise, the expression of Bcl-2 family member proteins are known to be tightly regulated by transcriptional and post-transcriptional modifications (33), and further efforts to elucidate the as yet unknown mechanisms of rapamycin regulation of $\mathrm{Bcl}-2$ expression is needed.

Our results suggested several potential therapeutic approaches for treating NSCLC with rapamycin. First, because our data indicate that the inhibition of mTOR induces apoptosis in both a PI3K- and AKT-independent manner, the development of a dual inhibitor of PI3K and mTOR (34), such as NVP-BEZ235 (35), may be more attractive alternative agents to inducing apoptosis in p53-mutated cancer cells than rapamycin alone. Second, since Bcl-2 has been revealed as the target of rapamycininduced apoptosis of NSCLC cell lines, Bcl-2 could be a novel molecular marker to select for cells sensitive to rapamycininduced apoptosis. Further studies are needed to address these issues.

In conclusion, rapamycin has potential activity to induce apoptosis in p53-mutated NSCLC cell lines, through downregulation of $\mathrm{Bcl}-2$ followed by cytochrome $\mathrm{c}$ release from mitochondria. These findings provide new insights into the possible antitumor mechanisms of rapamycin and may be useful in directing future dual inhibition therapy targeting the PI3K/ AKT pathway, as well as a novel biological marker for rapamycin sensitivity in lung cancer.

\section{Acknowledgements}

This study was supported by grants-in-aid for Scientific Research (C) 21590994 (to H. Chikumi and E. Shimizu) and 22590863 (to E. Shimizu and H. Chikumi) from the Ministry of Education, Science, and Culture, Sports, Science and Technology, Japan.

\section{References}

1. Jemal A, Siegel R, Ward E, Murray T, Xu J and Thun MJ: Cancer statistics, 2007. CA Cancer J Clin 57: 43-66, 2007.

2. Delbaldo C, Michiels S, Rolland E, et al: Second or third additional chemotherapy drug for non-small cell lung cancer in patients with advanced disease. Cochrane Database Syst Rev 17: CD004569, 2007.

3. Hanahan D: The hallmarks of cancer. Cell 100: 57-70, 2000.

4. Vignot S, Faivre S, Aguirre D and Raymond E: mTOR-targeted therapy of cancer with rapamycin derivatives. Ann Oncol 16: 525-537, 2005 .

5. Shaw RJ and Cantley LC: Ras, PI (3) K and mTOR signalling controls tumour cell growth. Nature 441: 424-430, 2006.
6. LoPiccolo J, Blumenthal GM, Bernstein WB and Dennis PA: Targeting the PI3K/Akt/mTOR pathway: effective combinations and clinical considerations. Drug Resist Updat 11: 32-50, 2008.

7. Sabers CJ, Martin MM, Brunn GJ, et al: Isolation of a protein target of the FKBP12-rapamycin complex in mammalian cells. J Biol Chem 270: 815-822, 1995.

8. Lorenz MC and Heitman J: TOR mutations confer rapamycin resistance by preventing interaction with FKBP12-rapamycin. J Biol Chem 270: 27531-27537, 1995.

9. Balsara BR, Pei J, Mitsuuchi Y, et al: Frequent activation of AKT in non-small cell lung carcinomas and preneoplastic bronchial lesions. Carcinogenesis 25: 2053-2059, 2004.

10. Wislez M, Spencer ML, Izzo JG, et al: Inhibition of mammalian target of rapamycin reverses alveolar epithelial neoplasia induced by oncogenic K-ras. Cancer Res 65: 3226-3235, 2005.

11. Marinov M, Fischer B and Arcaro A: Targeting mTOR signaling in lung cancer. Crit Rev Oncol Hematol 63: 172-182, 2007.

12. Gridelli C, Maione P and Rossi A: The potential role of mTOR inhibitors in non-small cell lung cancer. Oncologist 13: 139-147, 2008.

13. Muthukkumar S, RaMesh TM and Bondada S: Rapamycin, a potent immunosuppressive drug, causes programmed cell death in B lymphoma cells. Transplantation 60: 264-270, 1995.

14. Tirado OM, Mateo-Lozano S and Notario V: Rapamycin induces apoptosis of JN-DSRCT-1 cells by increasing the Bax: Bcl-xL ratio through concurrent mechanisms dependent and independent of its mTOR inhibitory activity. Oncogene 24: 3348-3357, 2005.

15. Zhang JF, Liu JJ, Lu MQ, et al: Rapamycin inhibits cell growth by induction of apoptosis on hepatocellular carcinoma cells in vitro. Transpl Immunol 17: 162-168, 2007.

16. Vega F, Medeiros LJ, Leventaki V, et al: Activation of mammalian target of rapamycin signaling pathway contributes to tumor cell survival in anaplastic lymphoma kinase-positive anaplastic large cell lymphoma. Cancer Res 66: 6589-6597, 2006.

17. Aguirre D, Boya P, Bellet D, et al: Bcl-2 and CCND1/CDK4 expression levels predict the cellular effects of mTOR inhibitors in human ovarian carcinoma. Apoptosis 9: 797-805, 2004.

18. Huang S, Liu LN, Hosoi H, Dilling MB, Shikata T and Houghton PJ: $\mathrm{p} 53 / \mathrm{p} 21^{\mathrm{CIP} 1}$ cooperate in enforcing rapamycin-induced G1 arrest and determine the cellular response to rapamycin. Cancer Res 61: 3373-3381, 2001.

19. Huang S, Shu L, Easton J, et al: Inhibition of mammalian target of rapamycin activates apoptosis signal-regulating kinase 1 signaling by suppressing protein phosphatase 5 activity. J Biol Chem 279: 36490-36496, 2004.

20. Calastretti A, Rancati F, Ceriani MC, Asnaghi L, Canti G and Nicolin A: Rapamycin increases the cellular concentration of the BCL-2 protein and exerts an anti-apoptotic effect. Eur J Cancer 37: 2121-2128, 2001

21. Boffa DJ, Luan F, Thomas D, et al: Rapamycin inhibits the growth and metastatic progression of non-small cell lung cancer. Clin Cancer Res 10: 293-300, 2004.

22. Buck E, Eyzaguirre A, Brown E, et al: Rapamycin synergizes with the epidermal growth factor receptor inhibitor erlotinib in non-small-cell lung, pancreatic, colon, and breast tumors. Mol Cancer Ther 5: 2676-2684, 2006.

23. Kishimoto M, Kohno T, Okudela K, et al: Mutations and deletions of the CBP gene in human lung cancer. Clin Cancer Res 11: 512-519, 2005.

24. Okabe T, Okamoto I, Tamura K, et al: Differential constitutive activation of the epidermal growthfactor receptor in non-small cell lung cancer cells bearing EGFR gene mutation and amplification. Cancer Res 67: 2046-2053, 2007.

25. Mori T, Okamoto H, Takahashi N, Ueda R and Okamoto T: Aberrant overexpression of 53BP2 mRNA in lung cancer cell lines. FEBS Lett 465: 124-128, 2000.

26. Park MJ, Shimizu K, Nakano T, et al: Pathogenetic and biologic significance of TP14ARF alterations in nonsmall cell lung carcinoma. Cancer Genet Cytogenet 141: 5-13, 2003.

27. Nagai Y and Miyazawa H: Genetic heterogeneity of the epidermal growth factor receptor in non-small cell lung cancer cell lines revealed by a rapid and sensitive detection system, the peptide nucleic acid-locked nucleic acid PCR clamp. Cancer Res 65: 7276-7282, 2005.

28. Kluck RM, Bossy-Wetzel E, Green DR and Newmeyer DD: The release of cytochrome $\mathrm{c}$ from mitochondria: a primary site for Bcl-2 regulation of apoptosis. Science 275: 1132-1136, 1997. 
29. del Peso L, Gonzalez-Garcia M, Page C, Herrera R and Nunez G: Interleukin-3-induced phosphorylation of BAD through the protein kinase Akt. Science 278: 687-689, 1997.

30. Gardai SJ, Hildeman DA, Frankel SK, et al: Phosphorylation of Bax Ser184 by Akt regulates its activity and apoptosis in neutrophils. J Biol Chem 279: 21085-21095, 2004.

31. Wangpaichitr M, Wu C, You M, et al: Inhibition of mTOR restores cisplatin sensitivity through down-regulation of growth and antiapoptotic proteins. Eur J Pharmacol 591: 124-127, 2008.

32. Migita K, Eguchi K, Ichinose Y, et al: Effects of rapamycin on apoptosis of rheumatoid synovial cells. Clin Exp Immunol 108: 199-203, 1997.
33. Gross A, McDonnell JM and Korsmeyer SJ: BCL-2 family members and the mitochondria in apoptosis. Genes Dev 13 1899-1911, 1999.

34. Zhang YJ, Duan Y and Zheng XF: Targeting the mTOR kinase domain: the second generation of mTOR inhibitors. Drug Discov Today 16: 325-331, 2011

35. Serra V, Markman B, Scaltriti M, et al: NVP-BEZ235, a dual $\mathrm{PI} 3 \mathrm{~K} / \mathrm{mTOR}$ inhibitor, prevents $\mathrm{PI} 3 \mathrm{~K}$ signaling and inhibits the growth of cancer cells with activating PI3K mutations. Cancer Res 68: $8022-8030,2008$ 